\title{
Toward the recovery of platinum group metals from a spent automotive catalyst with supported ionic liquid phases
}

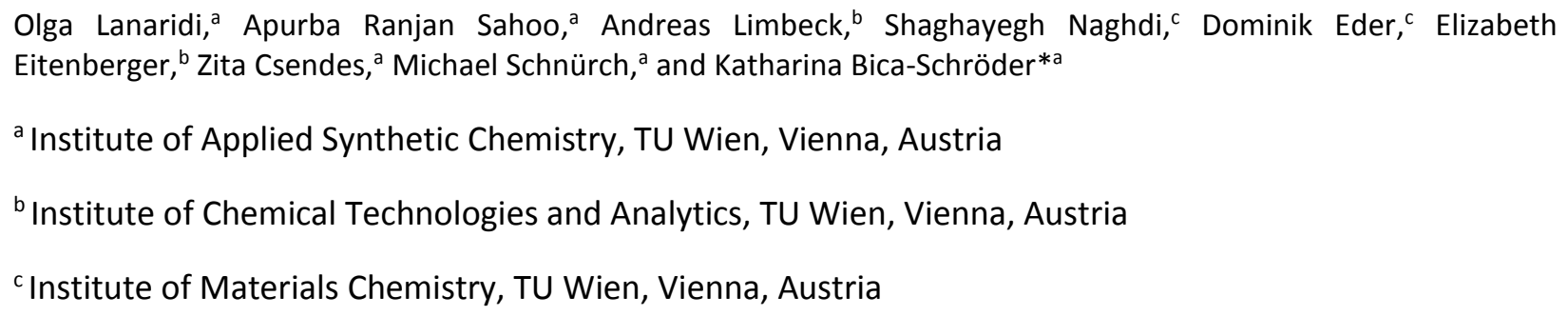

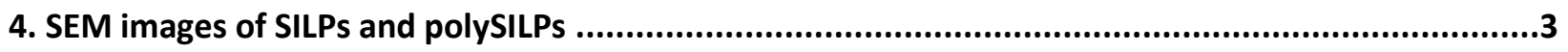

5. Retention of PGMs from model solutions on SILPs and polySILPs................................................7

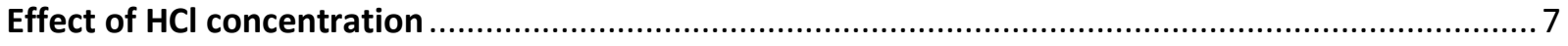

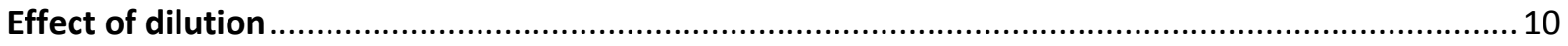

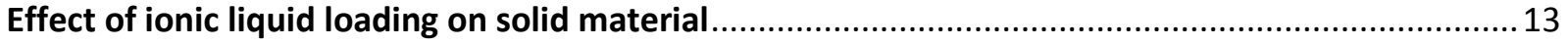

6. Retention of PGMs from real leachate on SILP 20\% (w/w) ......................................................13

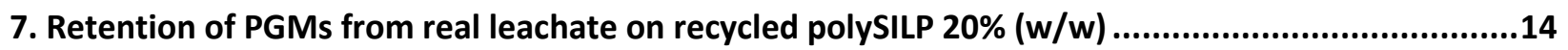

8. Breakthrough curves for polySILP 20\% (w/w) and Amberlite IRA-400.........................................14

9. Characterization of car catalyst material ....................................................................................15

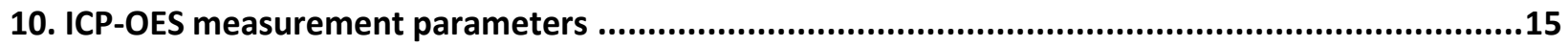




\section{Synthesis of SILP $P_{66614} \mathrm{Cl} 10 \%(w / w)$}

Silica 60 (Merck, size 40-60 $\mu \mathrm{m}$, specific surface area $480-540 \mathrm{~m}^{2} / \mathrm{g}$ ) was pre-dried in vacuum oven at 50 ${ }^{\circ} \mathrm{C}$ for $24 \mathrm{~h}$. A $250 \mathrm{ml}$ round bottom flask was charged with $23.0 \mathrm{~g}$ silica- 60 and $2.3 \mathrm{~g} \mathrm{P}_{66614} \mathrm{Cl}$. The mixture was suspended in $100 \mathrm{ml} \mathrm{CH} \mathrm{Cl}_{2}$ and shaken at room temperature until the complete amount of ionic liquid was dissolved. The solvent was removed carefully by reduced pressure. Remaining solvent traces were removed via high vacuum $\left(10^{-2} \mathrm{mbar}\right)$ for $24 \mathrm{~h}$. The procedure was accordingly adapted for the preparation of $20 \%$ and $50 \%$ loadings.

\section{Drift-IR spectra of SILPs}

The spectra were recorded with a Bruker Vertex 80FTIR spectrophotometer using a narrow band MCT (mercury-cadmium-telluride) detector measuring diffuse reflectance. 256 scans were collected for each spectrum with $4 \mathrm{~cm}^{-1}$ resolution.

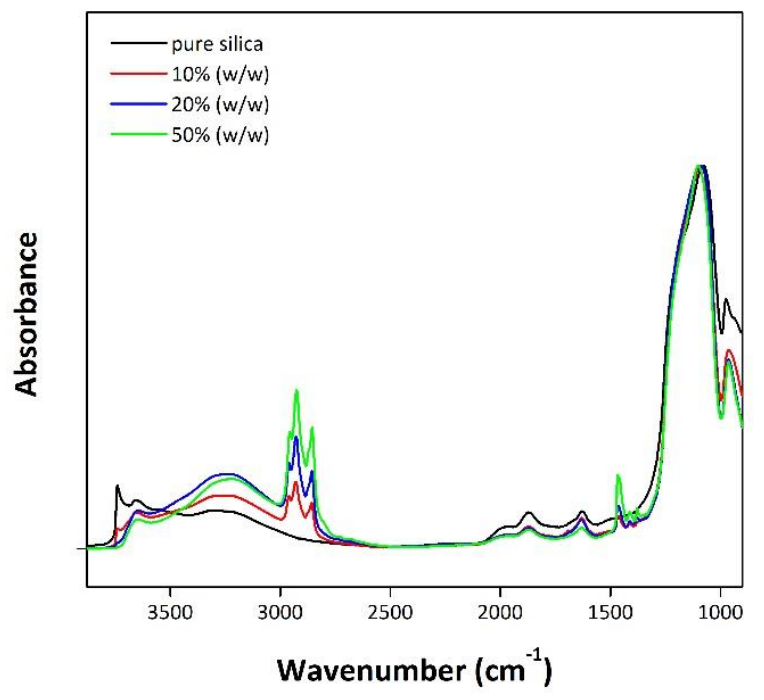

Figure S1 Drift-IR spectra of pure silica and SILPs with $\mathrm{P}_{66614} \mathrm{Cl}$ loadings of 10, 20 and 50\% (w/w).

\section{ATR-IR spectra of polySILPs}

Recorded with a Bruker Vertex 80FTIR spectrophotometer using a DTGS (deuterated triglycine sulfate) detector measuring diffuse reflectance. For each spectrum, 512 scans were collected with $4 \mathrm{~cm}^{-1}$ resolution. 


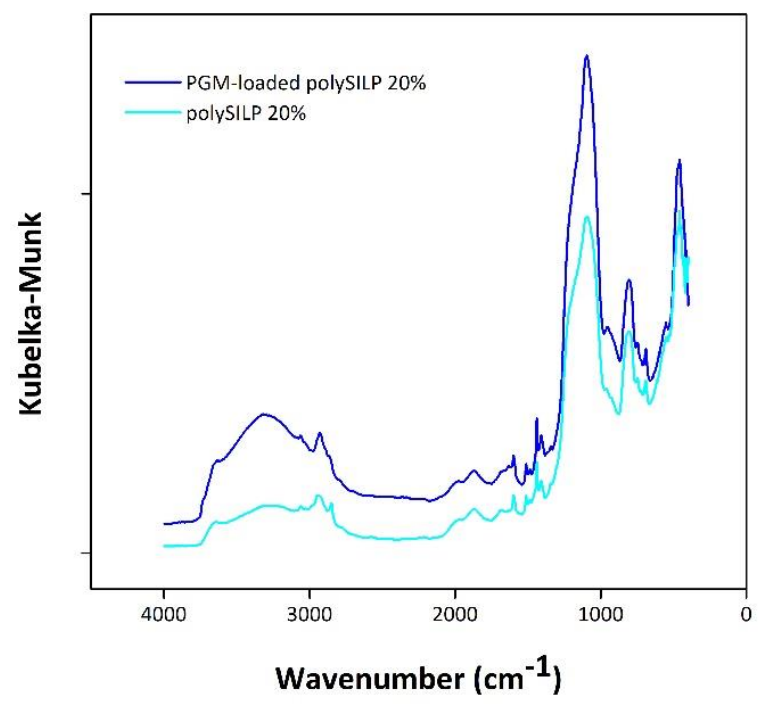

Figure S2 ATR-IR spectra of polySILP $20 \%$ and PGM-loaded polySILP $20 \%$.

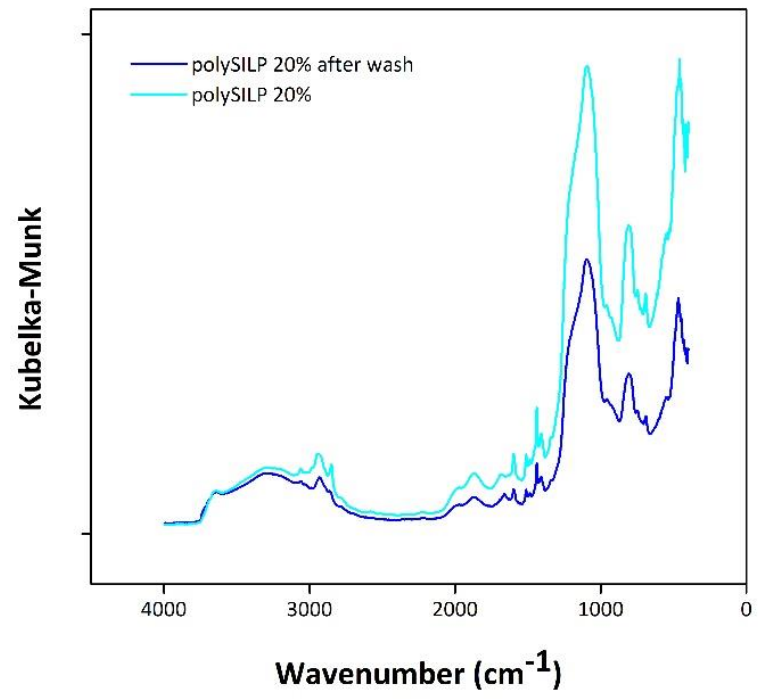

Figure S3 ATR-IR spectra of polySILP 20\% and used polySILP 20\% after washing with $0.1 \mathrm{M} \mathrm{HCl}$.

\section{SEM images of SILPs and polySILPS}

The images were recorded with a FEI Quanta 200 microscope equipped with an EDAX Octane Pro detector. Data were processed with the Team Enhanced software. 


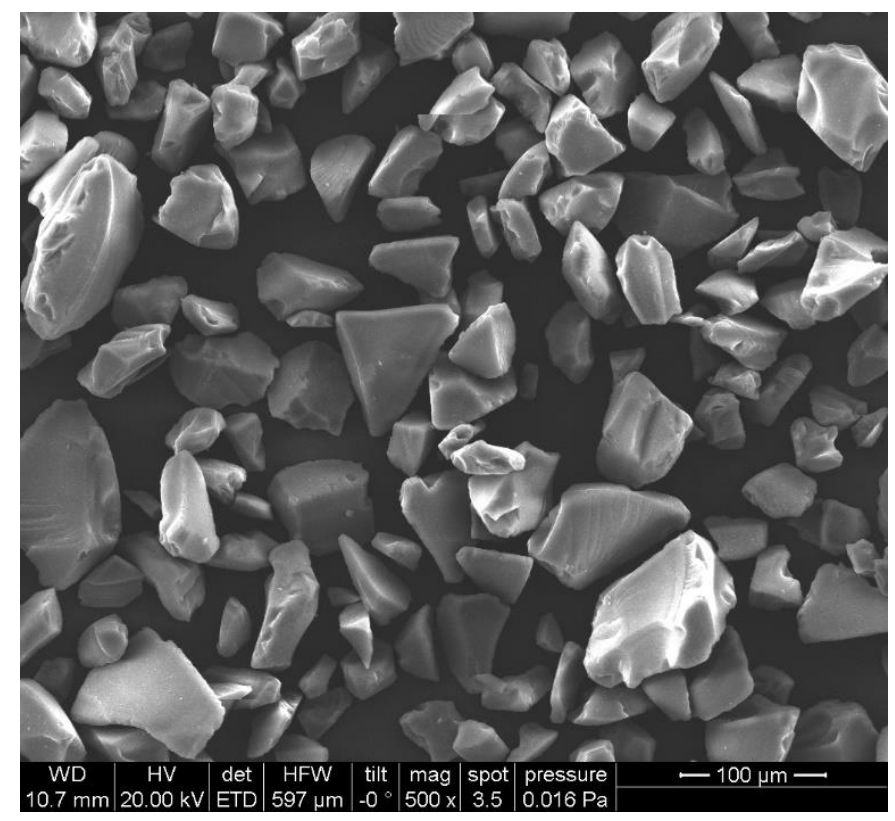

Figure S4 SEM image of SILP with $\mathrm{P}_{66614} \mathrm{Cl}$ loading of $20 \%(\mathrm{w} / \mathrm{w})$.

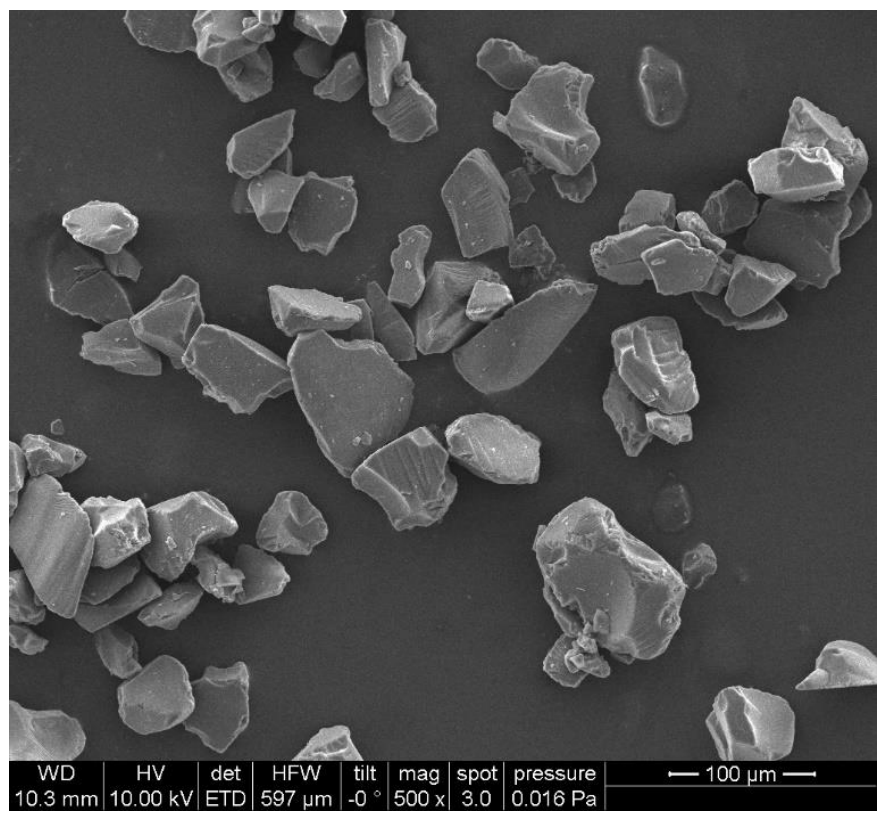

Figure S5 SEM image of PGM-loaded SILP with $\mathrm{P}_{66614} \mathrm{Cl}$ loading of $20 \%$ (w/w). 


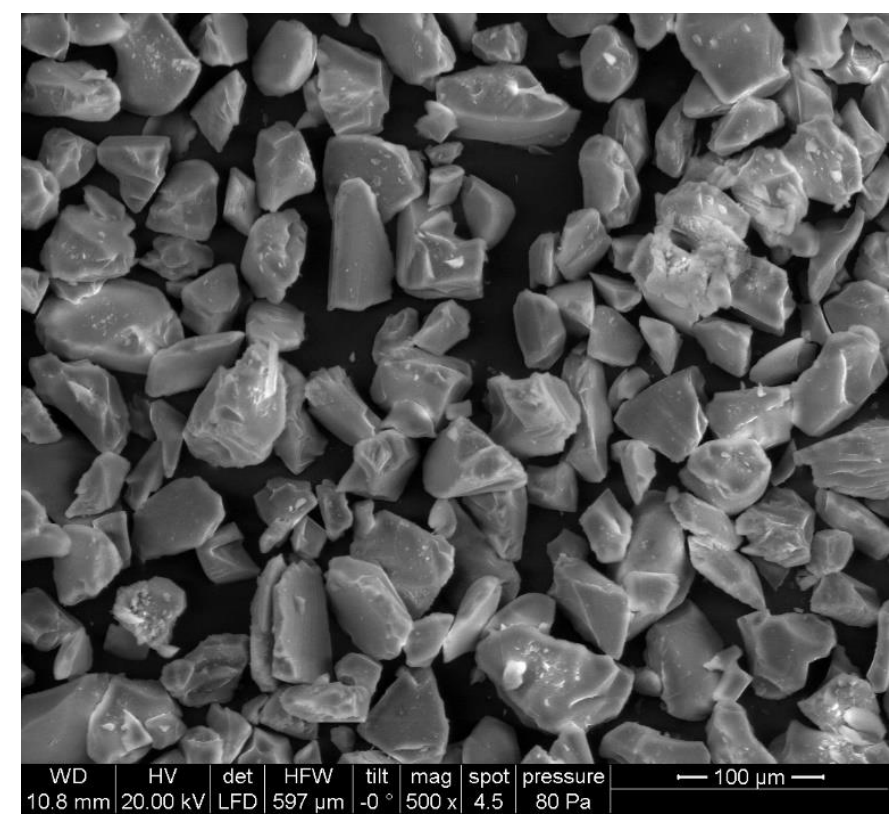

Figure S6 SEM image of polySILP with a loading of $20 \%(w / w)$.

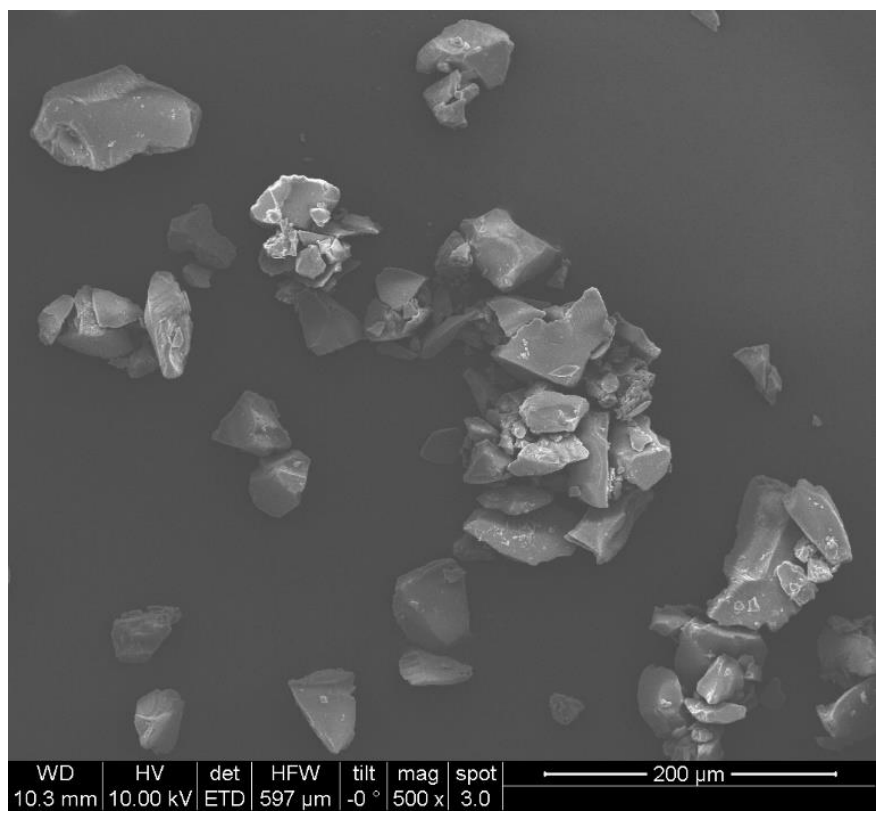

Figure S7 SEM image of PGM-loaded polySILP with a loading of $20 \%(w / w)$. 


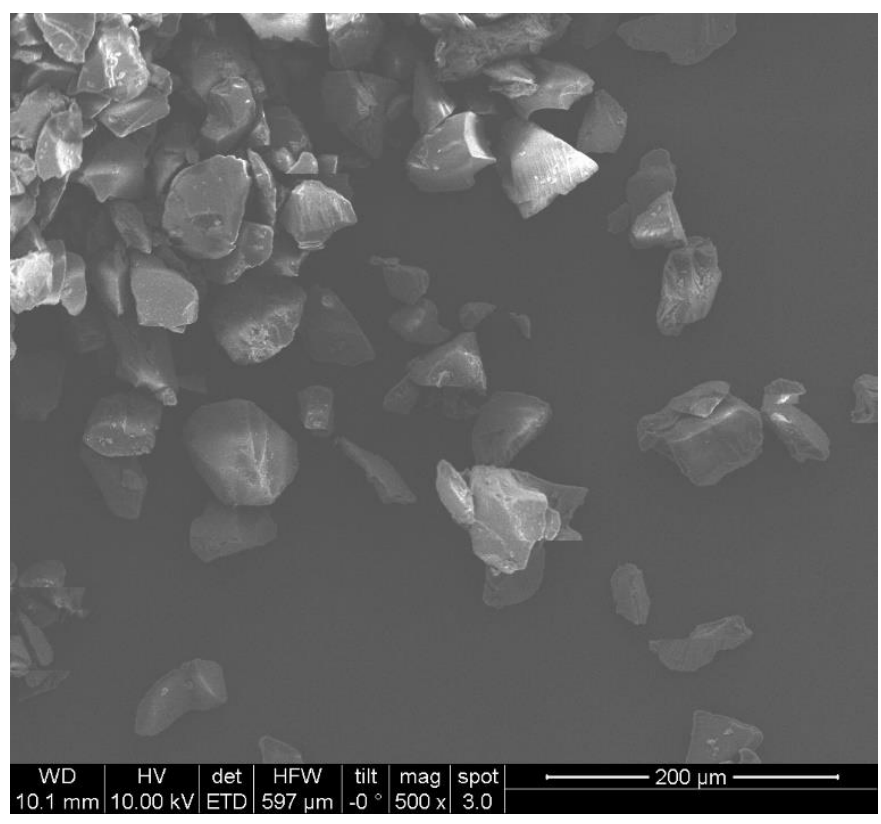

Figure S8 SEM image of polySILP with a loading of $20 \%(w / w)$ after stripping 1.

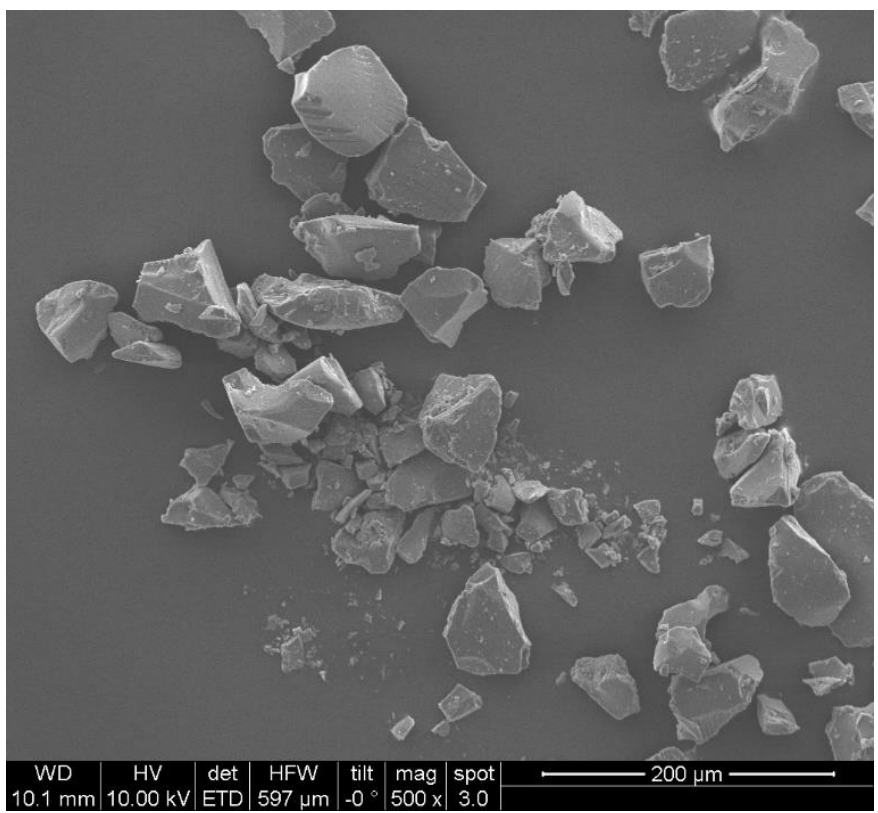

Figure S9 SEM image of polySILP with a loading of $20 \%(w / w)$ after stripping 2. 


\section{Retention of PGMs from model solutions on SILPs and polySILPs}

Effect of $\mathrm{HCl}$ concentration

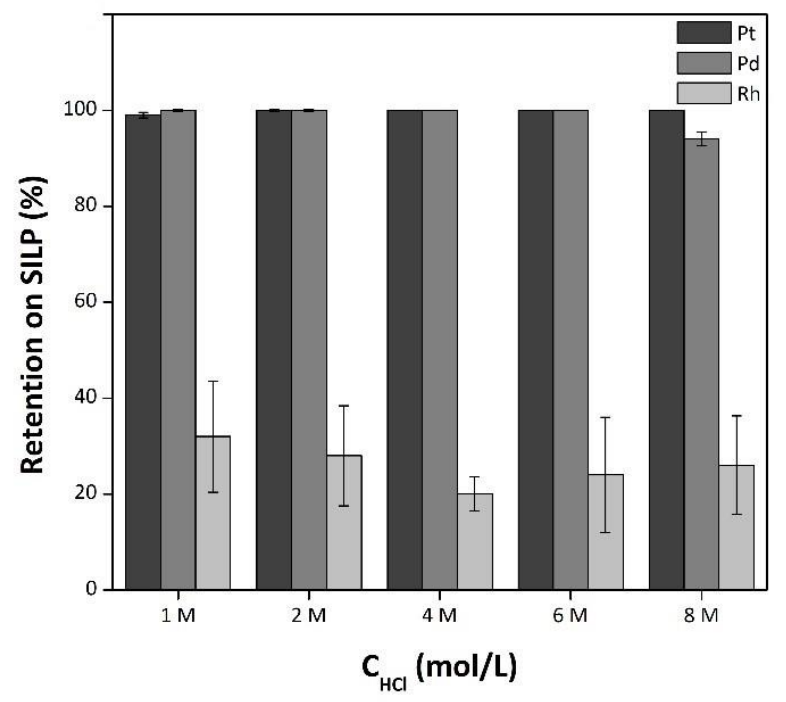

Figure S10 Effect of $\mathrm{HCl}$ concentration on PGM retention from model solution on SILP with $\mathrm{P}_{66614} \mathrm{Cl}$ loading of $10 \%$ (w/w).

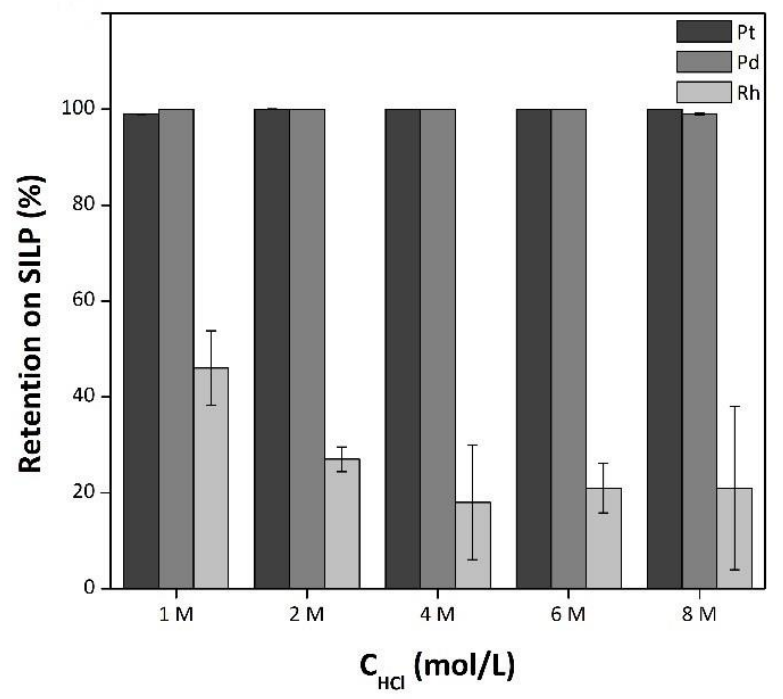

Figure S11 Effect of $\mathrm{HCl}$ concentration on PGM retention from model solution on SILP with $\mathrm{P}_{66614} \mathrm{Cl}$ loading of $20 \%$ (w/w). 


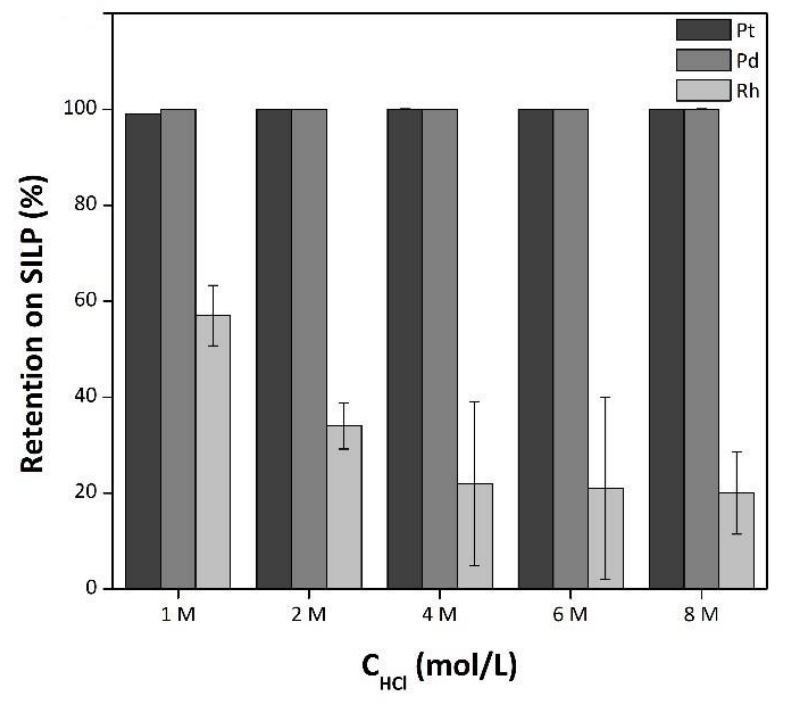

Figure S12 Effect of $\mathrm{HCl}$ concentration on PGM retention from model solution on SILP with $\mathrm{P}_{66614} \mathrm{Cl}$ loading of $50 \%$ (w/w).

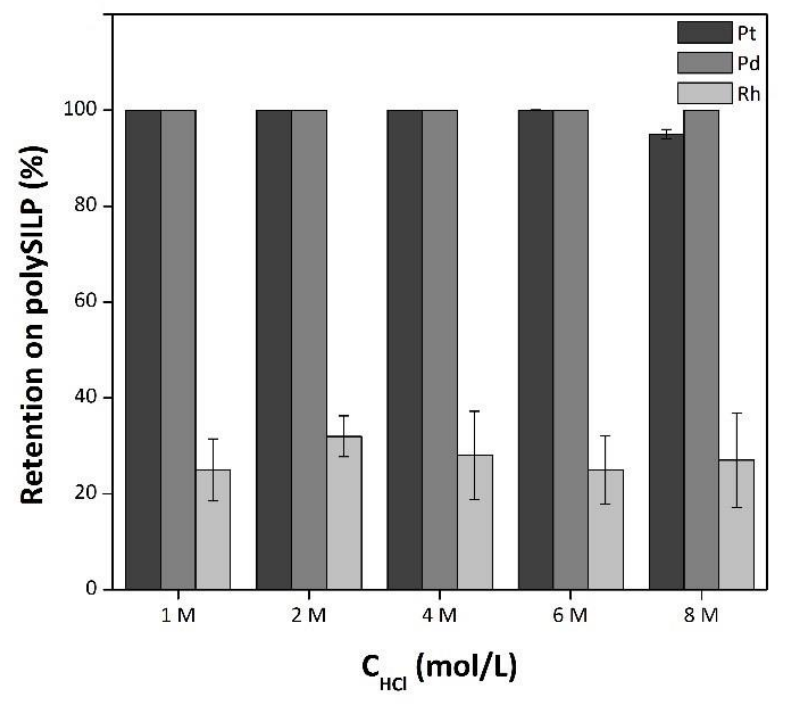

Figure S13 Effect of $\mathrm{HCl}$ concentration on PGM retention from model solution on polySILP with loading of $10 \%(w / w)$. 


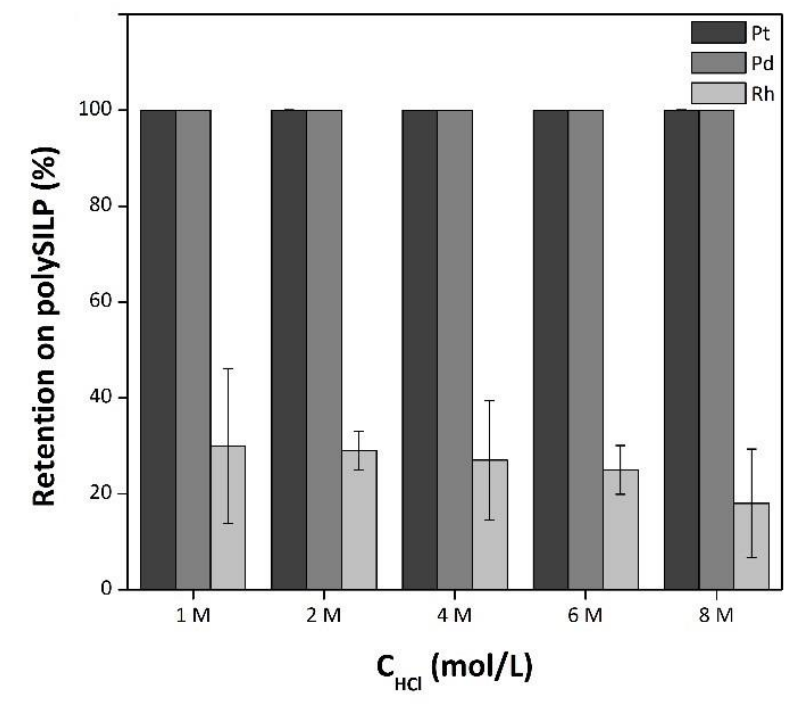

Figure S14 Effect of $\mathrm{HCl}$ concentration on PGM retention from model solution on polySILP with loading of $20 \%$ (w/w).

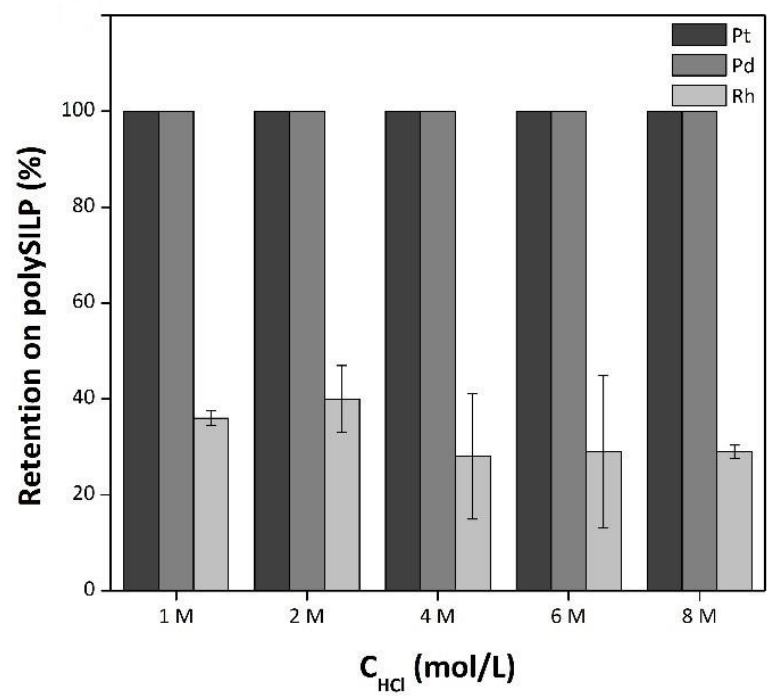

Figure S15 Effect of $\mathrm{HCl}$ concentration on PGM retention from model solution on polySILP with loading 50\% (w/w). 


\section{Effect of dilution}

The term "undiluted" refers to the model solution (194 ppm Pt, 318 ppm Pd and 34 ppm Rh) to contrast it with the different dilution levels.

The dilution was performed with $\mathrm{H}_{2} \mathrm{O}$ on a $\mathrm{X}: Y$ ratio, where $\mathrm{X}$ refers to the model solution and $\mathrm{Y}$ to $\mathrm{H}_{2} \mathrm{O}$.

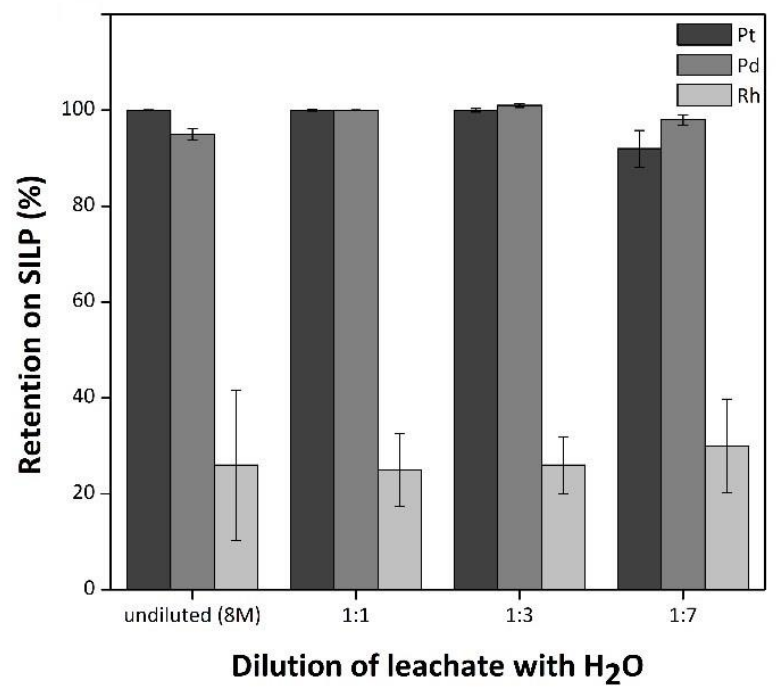

Figure S16 Effect of dilution PGM retention from model solution on SILP with $\mathrm{P}_{66614} \mathrm{Cl}$ loading of $10 \%(\mathrm{w} / \mathrm{w})$.

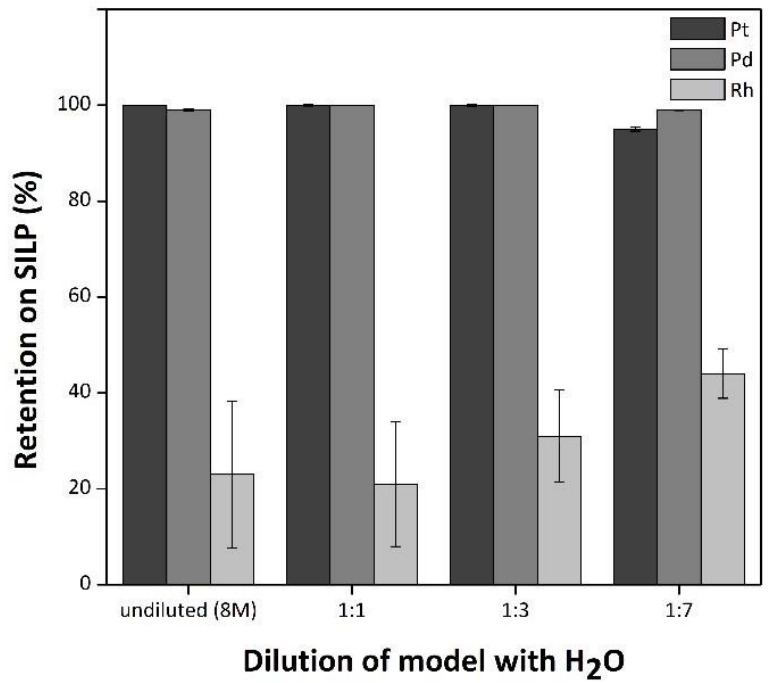

Figure S17 Effect of dilution on PGM retention from model solution on SILP with $\mathrm{P}_{66614} \mathrm{Cl}$ loading of $20 \%$ (w/w). 


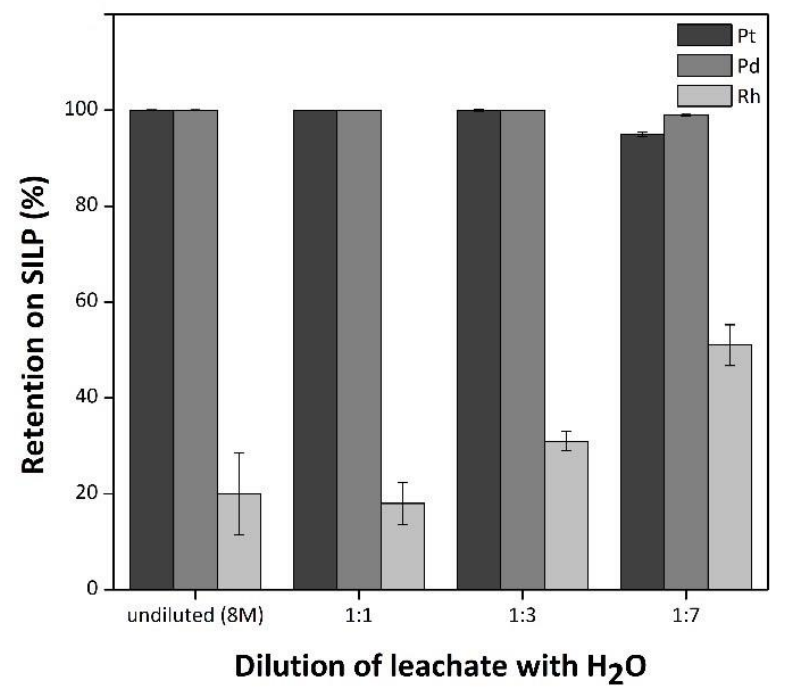

Figure S18 Effect dilution on PGM retention from model solution on SILP with $\mathrm{P}_{66614} \mathrm{Cl}$ loading of 50\% (w/w).

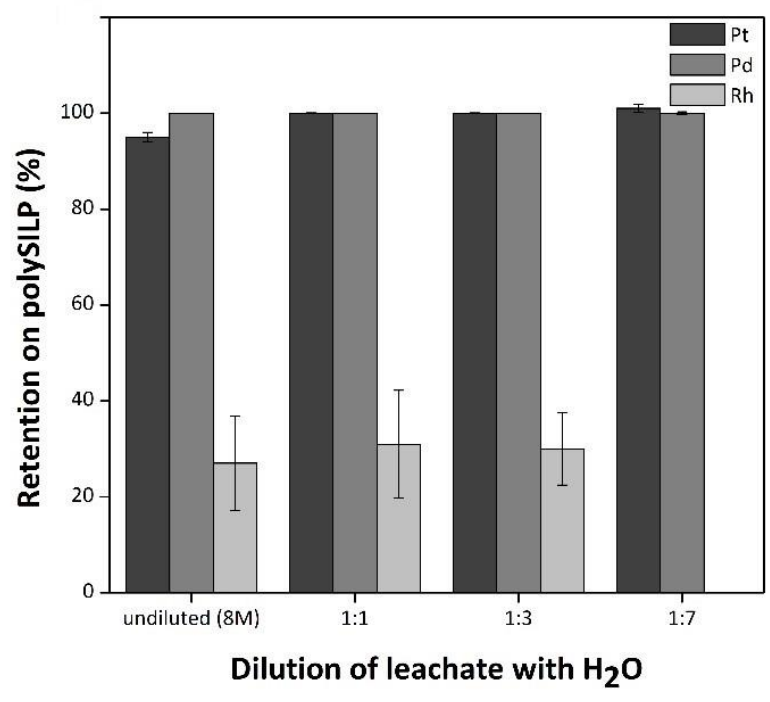

Figure S19 Effect of dilution on PGM retention from model solution on polySILP with loading of 10\% (w/w). 


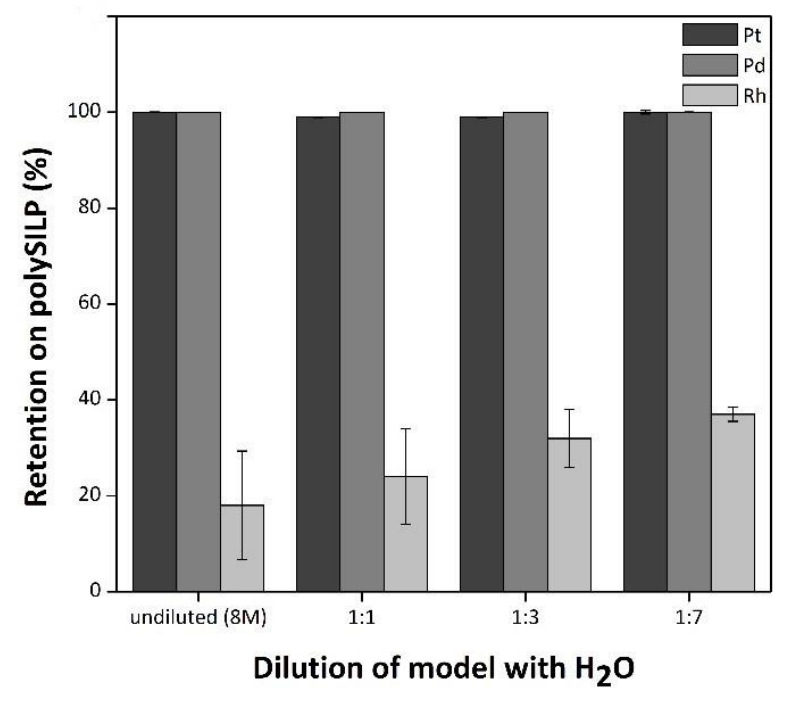

Figure S20 Effect of dilution on PGM retention from model solution on polySILP with loading of 20\% (w/w).

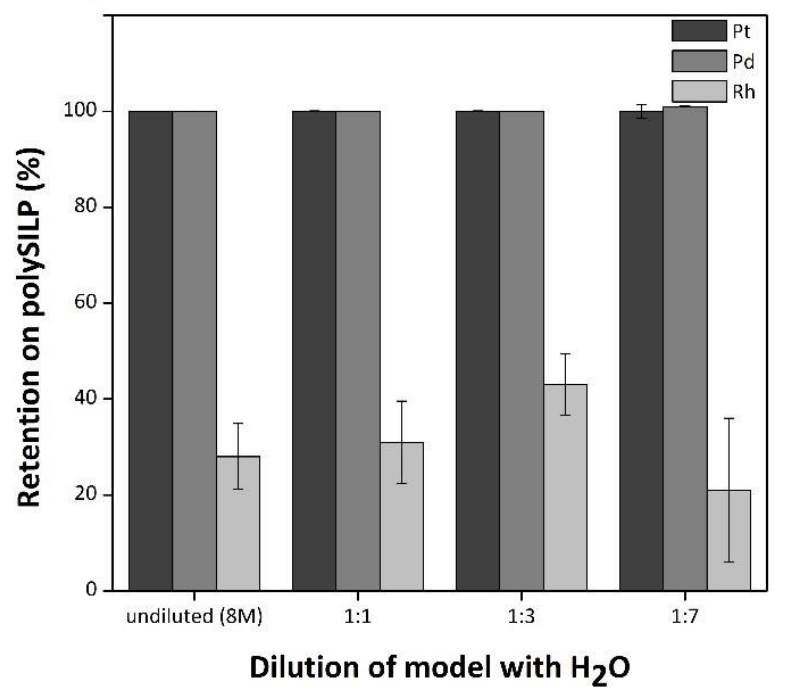

Figure S21 Effect of dilution on PGM retention from model solution on polySILP with loading of 50\% (w/w). 
Effect of ionic liquid loading on solid material

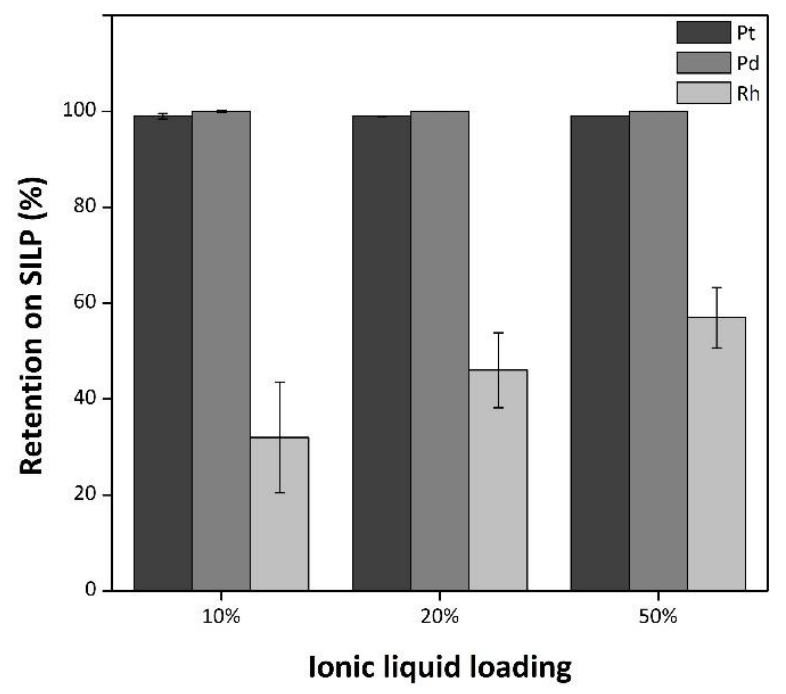

Figure S22 Effect of ionic liquid loading on SILP on PGM retention from 8M HCl model PGM solution diluted 1:7 with $\mathrm{H}_{2} \mathrm{O}$.

\section{Retention of PGMs from real leachate on SILP $20 \%(w / w)$}

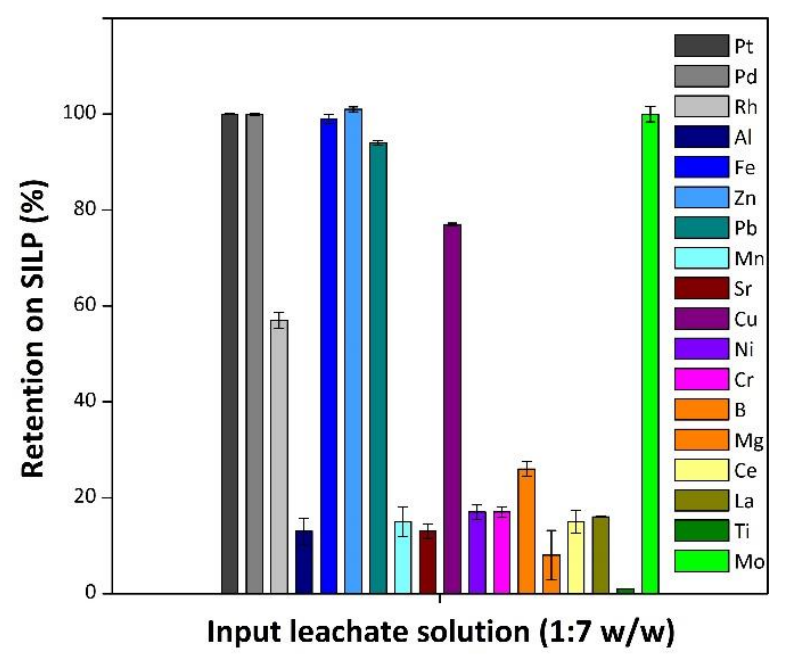

Figure S23 Retention behavior of PGMs and accompanying elements from automotive catalyst leachate on SILP 20\% (w/w). 


\section{Retention of PGMs from real leachate on recycled polySILP $20 \%(w / w)$}

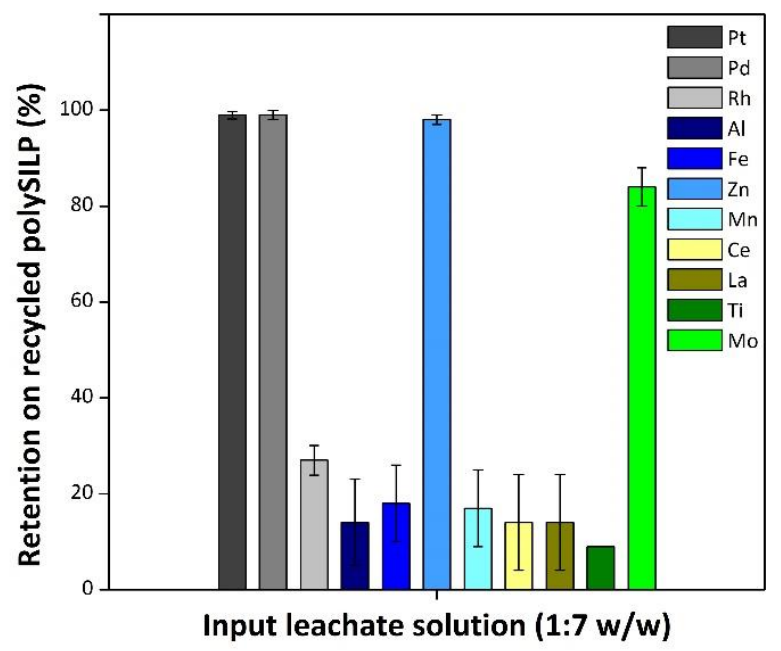

Figure S24 Retention behavior on recycled polySILP 20\% (w/w).

\section{Breakthrough curves for polySILP $20 \%(w / w)$ and Amberlite IRA-400}
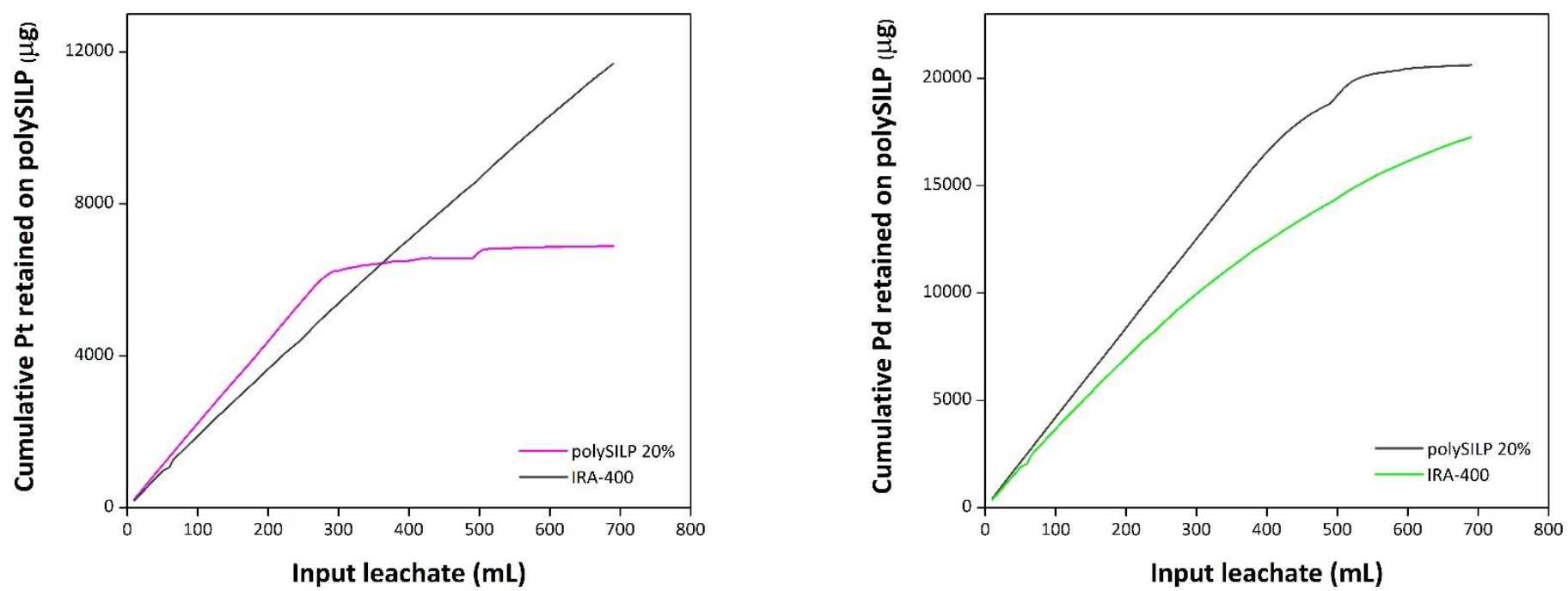

Figure S25 Breakthrough curves for Pt (left) and Pd (right) on polySILP 20\% (w/w) and Amberlite IRA-400. 


\section{Characterization of car catalyst material}

The spent car catalyst used in this study was fully characterized prior to the experiments (Table 1). It was digested with the aid of a mixture of mineral acids in a microwave oven (Multiwave 3000, Anton Paar, Germany), prior to measurement, for the complete dissolution of the ceramic material, which primarily comprises the car catalyst. The following digestion programme was employed; 8:00 at $500 \mathrm{~W}$; hold 8:00; 15:00 at $900 \mathrm{~W}$; hold 35:00. The quantification was performed by ICP-OES (Radial iCAP6500, Thermo Scientific, USA) with appropriate sample dilution. The fitness for purpose of the analytical measurement method was initially verified with the aid of the certified reference material ERM-EB504.

Table S1 Concentration of elements in car catalyst and acidic leachate

\begin{tabular}{ccc}
\hline Elements & $\begin{array}{c}\text { Solid catalyst } \\
\text { (ppm) }\end{array}$ & $\begin{array}{c}\text { Acidic leachate } \\
\text { (ppm) }\end{array}$ \\
\hline Pt & $970 \pm 20$ & $150 \pm 2$ \\
Pd & $1590 \pm 40$ & $293 \pm 4$ \\
Rh & $343 \pm 8$ & $27 \pm 0.4$ \\
Al & $311740 \pm 22722$ & $3512 \pm 38$ \\
Ce & $27600 \pm 1400$ & $1176 \pm 12$ \\
Fe & $7096 \pm 647$ & $655 \pm 8$ \\
Zn & $4226 \pm 574$ & $269 \pm 9$ \\
Sr & $830 \pm 225$ & $297 \pm 6$ \\
La & $3857 \pm 320$ & $384 \pm 4$ \\
Mg & $7687 \pm 309$ & $1036 \pm 14$ \\
Pb & $463 \pm 61$ & $69 \pm 5$ \\
Cu & $154 \pm 17$ & $20 \pm 0.4$ \\
Ni & $278 \pm 43$ & $13 \pm 0.6$ \\
Cr & $262 \pm 66$ & $23 \pm 0.2$ \\
Mn & $247 \pm 36$ & $17 \pm 0.3$ \\
Ba & $3165 \pm 770$ & $4 \pm 0.2$ \\
B & $65 \pm 10$ & $10 \pm 1$ \\
Ti & $3170 \pm 10$ & $47 \pm 1$ \\
Mo & $82 \pm 8$ & $15 \pm 0.2$ \\
\hline
\end{tabular}

\section{ICP-OES measurement parameters}

The PGM concentrations in all systems were quantified with a radial ICP-OES (Thermo iCAP 6500, Thermo Scientific, USA) with a sample introduction kit consisting of a parallel path nebulizer (PEEK Mira Mist, Thermo Scientific, Canada), a gas cyclonic spray chamber with a riser tube and a torch injector tube with $2 \mathrm{~mm}$ inner diameter. Signals in ICP-OES were recorded and processed using Qtegra 2.10 software (Thermo Scientific, USA).

Table S2 ICP-OES instrumental measurement parameters

\begin{tabular}{|l|l|}
\hline Instrument & Radial iCAP 6500 (Thermo Scientific, USA) \\
\hline Software & Qtegra 2.10 (Thermo Scientific, USA) \\
\hline RF power & $1400 \mathrm{~W}$ \\
\hline Torch internal diameter & $2.0 \mathrm{~mm}$ \\
\hline
\end{tabular}




\begin{tabular}{|l|l|}
\hline Nebulizer & V-groove nebulizer (Thermo Scientific, USA) \\
\hline Nebulizer gas flow & $0.70 \mathrm{~L} / \mathrm{min}$ \\
\hline Coolant gas flow & $12 \mathrm{~L} / \mathrm{min}$ \\
\hline Auxiliary gas flow & $0.80 \mathrm{~L} / \mathrm{min}$ \\
\hline Sample introduction flow rate & $0.70 \mathrm{~mL} / \mathrm{min}$ \\
\hline Viewing height & $10 \mathrm{~mm}$ \\
\hline Exposure time (in Vis) & $10 \mathrm{sec}$ \\
\hline Observation time per measurement & $5 \mathrm{sec}$ \\
\hline Number of replicates pre measurement & 5 \\
\hline
\end{tabular}

Table S3 Selected emission wavelengths for elemental quantification

\begin{tabular}{|c|c|c|}
\hline Element & Quantification wavelength & Quality control wavelength \\
\hline $\mathrm{Pt}$ & 265.945 & 214.423 \\
\hline $\mathrm{Pd}$ & 340.458 & 324.270 \\
\hline $\mathrm{Rh}$ & 343.489 & 369.236 \\
\hline $\mathrm{Al}$ & 309.271 & 237.312 \\
\hline $\mathrm{Fe}$ & 259.940 & 238.204 \\
\hline $\mathrm{Zn}$ & 213.856 & 202.548 \\
\hline $\mathrm{Sr}$ & 407.771 & 421.552 \\
\hline $\mathrm{Mg}$ & 279.553 & 280.270 \\
\hline $\mathrm{Ca}$ & 315.887 & 318.128 \\
\hline $\mathrm{Pb}$ & 220.353 & 216.999 \\
\hline $\mathrm{Ce}$ & 456.236 & 380.152 \\
\hline $\mathrm{Cu}$ & 324.754 & 327.396 \\
\hline $\mathrm{Ni}$ & 221.647 & 231.604 \\
\hline $\mathrm{Mn}$ & 257.610 & 293.930 \\
\hline $\mathrm{Cr}$ & 267.716 & - \\
\hline $\mathrm{Ba}$ & 455.403 & - \\
\hline $\mathrm{B}$ & 249.773 & 249.678 \\
\hline $\mathrm{Mo}$ & 202.030 & - \\
\hline $\mathrm{La}$ & 261.034 & - \\
\hline $\mathrm{Ti}$ & 334.941 & $308.802-$ \\
\hline & & \\
\hline
\end{tabular}

